\title{
Vesicoureteric reflux and timing of micturating cystourethrography after urinary tract infection
}

Jonathan C Craig, John F Knight, Premala Sureshkumar, Albert Lam, Ella Onikul, L Paul Roy

\begin{abstract}
Objective-To test the medical belief that the micturating cystourethrogram (MCU) be deferred four to six weeks after acute symptomatic urinary tract infection (UTI) because of the risk of falsely detecting vesicoureteric reflux if performed earlier.

Study design-A cross sectional analytic study of preschool children with first time symptomatic UTI.

Results-Of the 284 eligible children, 272 $(95.8 \%)$ had MCU at a median time of 29 days after diagnosis (range 5 to 167 days). Vesicoureteric reflux was present in 77 children $(28.3 \%)$. Beyond one week after diagnosis ( 270 children) the proportion and severity of vesicoureteric reflux detected was not associated with the timing of the MCU. Before one week, both children tested had vesicoureteric reflux. Conclusions-The presence and grade of vesicoureteric reflux is not influenced by the timing of the MCU one week after acute symptomatic UTI. There may be an association between the MCU and the presence of vesicoureteric reflux for children tested within one week after UTI. The MCU need not be deferred for four to six weeks after UTI.

(Arch Dis Child 1997;76:275-277)
\end{abstract}

Keywords: urinary tract infection; micturating cystourethrogram; vesicoureteric reflux.

By 7 years of age $8 \%$ of girls and $2 \%$ of boys can be expected to develop at least one symptomatic urinary tract infection. ${ }^{1}$ As vesicoureteric reflux has been documented in one third to one half of children with urinary tract infection, ${ }^{2-4}$ a micturating cystourethrogram (MCU) is often recommended for the investigation of these children. Current standard paediatric, ${ }^{56}$ paediatric nephrology, ${ }^{78}$ and paediatric urology ${ }^{9}$ textbooks recommend that the MCU be performed four to six weeks after starting effective treatment. This recommendation is made on the grounds that urine infection with inflammation surrounding the vesicoureteric junction may cause transient vesicoureteric reflux, or increase the severity of the baseline vesicoureteric reflux. However, there are no human studies which support this teaching. This four to six week waiting time is not without the potential for harm and cost: antibiotics are given daily to prevent recurrence of infection, and there is parental anxiety surrounding the uncertainty of the diagnosis of vesicoureteric reflux until the MCU is performed. The MCU could also be more conveniently arranged earlier when other imaging procedures are performed, such as renal tract sonography. To determine whether there is an association between the timing of the MCU after urinary tract infection and the presence and severity of vesicoureteric reflux, we carried out a cross sectional study using the entry data of a large cohort of consecutive preschool children who had a MCU as part of their assessment for a first symptomatic urinary tract infection.

\section{Methods}

Consecutive children under 5 years presenting to the emergency department of the Royal Alexandra Hospital for Children, Sydney, between March 1993 and December 1994 with a symptomatic urinary tract infection were identified prospectively through the hospital laboratory and were enrolled in a cohort study. All urine samples were inoculated onto blood agar and MacConkey medium using a $1 \mu \mathrm{l}$ calibrated loop. For this study, symptomatic urinary tract infection was defined as a colony forming unit count of $>10^{6} / 1$ from urine obtained from a suprapubic tap or catheter sample, or $>10^{7} / 1$ from a mid stream voided urine sample, in combination with symptoms or signs of urinary tract infection. Children with a previous diagnosis of urinary tract infection, or a known renal tract, neurological, or skeletal predisposing cause were excluded. A detailed medical history and examination were conducted by one investigator (JCC) and the results recorded before any renal tract imaging.

The MCU was performed using $50-250 \mathrm{ml}$ of urograffin $30 \%$, which was instilled into the bladder through a paediatric feeding tube by gravity until voiding occurred. The antegrade passage of contrast through the urethra and retrograde passage of contrast up the ureter (vesicoureteric reflux) were observed with fluoroscopy. Baseline static images, two oblique bladder views, early, mid, and late voiding views, a post-void picture of the bladder to document residual bladder volume, and a film of the kidneys to document the degree of reflux were obtained.

Children were either on treatment or on prophylactic doses of antibiotics at the time of testing. Sedation was not given and urine samples for culture were not routinely obtained at the time. The MCU was arranged at the discretion of the attending physician-usually 
Table 1 Frequency and grade of vesicoureteric reflux (VUR) (children)

\begin{tabular}{lll}
\hline Grade of VUR & Frequency & $\%$ \\
\hline Nil & 195 & 71.7 \\
Grade I & 14 & 5.1 \\
Grade II & 28 & 10.3 \\
Grade III & 19 & 7.0 \\
Grade IV & 12 & 4.4 \\
Grade V & 4 & 1.5 \\
Total & 272 & 100.0 \\
\hline
\end{tabular}

according to availability of appointments - to coincide with renal sonography, and so that all renal tract imaging would be performed before clinical review of the child six weeks after presentation.

All MCUs were reported by one of two paediatric radiologists and graded according to the international study of reflux in children. ${ }^{10}$ If reflux was bilateral and unequal the patient was assigned according to the higher grade of reflux. The radiologists were blinded regarding the timing of the MCU relative to the UTI and to the study hypothesis.

The timing of the MCU after the urinary tract infection was calculated by the time difference between start of antibiotic treatment after diagnosis and the performance of the test. The presence and grade of vesicoureteric reflux detected in each week after the infection (weeks $0-7+)$ were compared using the $\chi^{2}$ and exact tests for association and trend. A significance level of 0.05 was used. Because the MCU was not performed at random intervals after the infection, potential factors that may have been associated with vesicoureteric reflux or the decision on timing of the MCU were adjusted for using logistic regression, with the presence of vesicoureteric reflux modelled as the outcome variable.

Institutional ethics approval was obtained for the study, and informed consent was obtained from the parents of the children who were study subjects.

\section{Results}

During the study period 284 children fulfilled the entry criteria; 272 children $(95.8 \%)$ had an MCU performed; 12 children $(4.2 \%)$ did not participate because of parental refusal.

There were 149 boys (54.8\%) and 123 girls $(45.2 \%)$. The median age was 8.3 months (range 0.3 to 59.9 months). Vesicoureteric reflux was detected in 77 children (table 1). Bladder outlet obstruction secondary to posterior urethral valves was present in one child and intrarenal reflux was detected in seven $(2.6 \%)$. No child developed a symptomatic iatrogenic urinary tract infection after the MCU.

The timing of the MCU, and the grade and presence of vesicoureteric reflux detected are given in table 2 . The median time between diagnosis and the MCU was 29 days (interquartile range 23-34 days, range 5-167 days).

Overall, there was evidence of a weak association between timing of the MCU and grade of vesicoureteric reflux (table $2: \chi_{(14)}^{2}=24.8, \mathrm{p}=$ $0.04)$. This was not because of a trend towards increased detection and severity of reflux with decreasing time between diagnosis of urinary tract infection and the $\operatorname{MCU}\left(\chi_{(1)}^{2}=1.3, \mathrm{p}=\right.$ $0.3)$. Rather, the positive association was because of two cases of grade III-V vesicoureteric reflux in children who had the MCU performed within the first week after diagnosis (Fisher's exact test; $\mathrm{p}=0.02$ ).

Using logistic regression, after adjustment for factors that may have been associated with the presence of vesicoureteric reflux or may have influenced the timing of the MCU-age, gender, a history of fever, vomiting, lethargy, anorexia, diarrhoea, duration of illness, a past history of previous unexplained fever, recorded temperature on admission-there was no evidence of an association between the timing of the MCU after urinary tract infection and vesicoureteric reflux $\left(\chi^{2}=0.58, p=0.5\right.$; odds ratio $0.93,95 \%$ confidence interval 0.76 to 1.12).

\section{Discussion}

Despite the common nature of urinary tract infection and the frequency with which MCU is performed in children, we could not find any studies in children which have analysed the association between timing of the MCU after urinary tract infection and the detection and grade of vesicoureteric reflux. The frequency and grade of vesicoureteric reflux observed in this study was similar to previous studies..$^{2-4}$ Beyond one week after the diagnosis of urinary tract infection there was no evidence of an association between the presence or grade of vesicoureteric reflux and timing of the MCU, even after adjustment for factors that may predict for reflux or clinical indicators that may have influenced the timing of the MCU. The children who had the MCU within one week of diagnosis, however, were more likely to show vesicoureteric reflux, and of a high grade, but because there were only two patients in the early MCU group, we should be cautious not to overinterpret this finding. There may have been other factors which prompted the early MCU in these children which would have made vesicoureteric reflux more likely, but which we could not adjust for in this study. These data may support the notion that acute inflammation does cause transient malfunction of the vesicoureteric junction.

MCU after urinary tract infection appears to be a very safe procedure: there were no episodes of symptomatic infection following the test. Concerns regarding ascending spread of infection may be unfounded as long as appropriate chemoprophylactic cover is provided.

The present study did not involve a random allocation of timing of the MCU and so there is a potential for bias. Apart from randomising the time of the MCU, an ideal study may have involved performing the test on each child at two time intervals after diagnosis-early and late-and comparing proportions in a paired manner. We did not think such a study was feasible because of the invasive nature of even a single MCU. Measurement error has been minimised by blinding the radiologists to the study hypothesis and to the time of infection. 
Table 2 Grade of vesicoureteric reflux (VUR) detected and timing of micturating cystourethrogram (MCU)

\begin{tabular}{|c|c|c|c|c|c|c|}
\hline \multirow{2}{*}{$\begin{array}{l}\text { Time from UTI } \\
\text { (days) }\end{array}$} & \multirow{2}{*}{$\begin{array}{l}\text { No of children } \\
\text { tested }\end{array}$} & \multicolumn{3}{|c|}{ Grade of VUR } & \multicolumn{2}{|c|}{ All VUR } \\
\hline & & 0 & $I-I I$ & $I I I-V$ & No & $\%$ \\
\hline $0-6$ & 2 & 0 & 0 & 2 & 2 & 100.0 \\
\hline $7-13$ & 20 & 17 & 0 & 3 & 3 & 15.0 \\
\hline $14-20$ & 38 & 23 & 10 & 5 & 15 & 39.4 \\
\hline $21-27$ & 60 & 45 & 8 & 7 & 15 & 25.0 \\
\hline $28-34$ & 85 & 60 & 12 & 13 & 25 & 39.4 \\
\hline $35-41$ & 26 & 19 & 5 & 2 & 7 & 36.9 \\
\hline $42-48$ & 17 & 14 & 3 & 0 & 3 & 17.6 \\
\hline $49+$ & 24 & 17 & 4 & 3 & 7 & 29.2 \\
\hline
\end{tabular}

UTI = urinary tract infection.
$0.66, \mathrm{p}=0.4)$. Although the study does have limitations the data do suggest that active infection is not associated with vesicoureteric reflux.

Deferring the MCU for four to six weeks after urinary tract infection is widely recommended because of a proposed association between the timing of the MCU after the infection and the detection of vesicoureteric reflux in children. We could find no evidence of this association beyond one week after infection. An MCU within one week of infection may be more likely to demonstrate vesicoureteric reflux. Acute infective complications did not occur. Deferring the MCU for four to six weeks after infection carries inevitable cost-parents are worried about the presence of reflux and children are on daily antibiotics. This study suggests these costs are not outweighed by the unconfirmed concern for falsely detecting vesicoureteric reflux, provided the MCU is delayed one week after urinary tract infection. We conclude that the MCU can be arranged at any time after the first week of diagnosis according to convenience considerations rather than after four to six weeks, as is commonly recommended. produced using (inoculation of bacteria through nephrostomy tubes, ${ }^{11}{ }^{12}$ submucosal insertion of bacteria impregnated agar and bladder trauma, ${ }^{13}$ inoculation of bacteria into a renal artery, ${ }^{13}$ or periurethral inoculation with instillation of a foreign body into the bladder ${ }^{14}$ ) and reflux was subsequently demonstrated with variable success. Later, Roberts and Riopelle ${ }^{15}$ compared the rate of resolution of reflux in 16 infant female rhesus monkeys. Eight were instilled with coliform bacteria at the time of monthly cystography, and the control group were not. They concluded that vesicoureteric reflux took longer to resolve in the infected group, even though the mean time that reflux was present was not significantly different between the two groups. It is reasonably well established that the introduction of bacteria into the ureter can cause ureteritis and abnormal peristaltic activity in animals ${ }^{16}$; however, it remains uncertain whether transient urinary infection alone can cause vesicoureteric reflux in animals. Extrapolating these data to children who do not have artificial manipulation of their renal tracts and have transient infection does involve a number of unproven assumptions.

To our knowledge there has been only one clinical study published in this area. The study of Gross and Lebowitz ${ }^{17}$ addressed a related but slightly different question: are children with urinary tract infection at the time of the MCU more likely to have vesicoureteric reflux than those with sterile urine? They reviewed a case series of children who had an MCU performed within their unit over a one year period. The proportion of children with infected urine at the time of the MCU was no different in children with or without reflux (25/ 204 with reflux, $40 / 397$ without reflux, $\chi_{(1)}^{2}=$
This study was supported by a National Health and Medical Research Project Grant. Financial assistance was also given by the Manildra Group of Companies. We thank Geoffrey Berry for statistical advice, and Les Irwig and Elisabeth Hodson for comments on earlier drafts.

1 Hellstrom A, Hanson E, Hansson S, Hialmas K, Jodal U. Association between urinary symptoms at 7 years old and
previous urinary tract infection. Arch Dis Child previous urinary

2 Middleton AW, Nixon GW. The lack of correlation between upper tract changes on excretory urography and significant vesicoureteral reflux. $\mathcal{F}$ Urol 1980;123:227-8.

3 Smellie JM, Normand ICS, Katz G. Children with urinary tract infection: a comparison of those with and those without vesicoureteric reflux. Kidney Int 1981;20:717-22. early radiological investigations of urinary tract infection in children. Lancet 1974;ii:403-4.

5 Travis LB. The kidneys and urinary tract. In: Rudolph AM, ed. Pediatrics. 19th Ed. Norwalk: Appleton and Lange, 1991:1223-308. In: Behrman RE, ed. Nelson textbook of pediatrics. 14th Ed. Philadelphia: WB Saunders, 1992:1323-92.

7 Rushton HG Belman AB. Vesicoureteral reflux and renal scarring. In: Holliday MA, Barratt TM, Avner ED, eds. Pediatric nephrology. 3rd Ed. Baltimore: Williams and Pediatric nephrology.

8 Swischuk LE, Hayder CK. Imaging of the urinary tract. In: Edelmann CM, ed. Pediatric kidney disease. 2nd Ed. Boston: Edelmann CM, ed. Pediatric kidney disease. 2nd

9 Kramer SA. Vesicoureteric reflux. In: Kelalis PP, King LR, Belman AB, eds. Clinical pediatric urology. 3rd Ed. Philadelphia: WB Saunders, 1992:441-99.

10 International Reflux Study in Children. International system of radiographic grading of vesicoureteric reflux. Pediatr Radiol 1985;15:105-9.

11 Schoenberg HW, Beisswanger P, Howard WJ, Klingenmaier $\mathrm{H}$, Walter CF, Murphy JJ. Effect of lower urinary tract infection upon ureteral function. F Urol 1964;92:107-8.

12 Kaveggia L, King LR, Grana L, Idriss FS. Pyelonephritis: a cause of vesicoureteral reflux? f Urol 1966;95:158-63.

13 Jeffs RD, Allen MS. The relationship between ureterovesical reflux and infection. F Urol 1962;88:691-5.

14 Sommer JL, Roberts JA. Ureteral reflux resulting from chronic urinary infection in dogs: long-term studies. F Urol 1966;95:502-10.

15 Roberts JA, Riopelle AJ. Vesicoureteric reflux in the primate: ureterovesical junction. Pediatrics 1978;61:853-7.

16 Roberts JA. Experimental pyelonephritis in the monkey: IV. Vesicoureteral reflux and bacteria. Invest Urol 1976;14:198.

17 Gross GW, Lebowitz RL. Infection does not cause reflux. Am $\mathcal{F}$ Radiol 1981;137:929-32.
4 Saxena SR, Laurance BM, Shaw DG. The justification for

6 Bergstein JM. The urinary system and pediatric gynecology. III. Effect of urinary tract infection on maturation of the 